\title{
The strongly distance-balanced property of the generalized Petersen graphs*
}

\author{
Klavdija Kutnar \\ University of Primorska, FAMNIT, Glagoljaška 8, 6000 Koper, Slovenia \\ Aleksander Malnič \\ University of Ljubljana, IMFM, Jadranska 19, 1111 Ljubljana, Slovenia \\ Dragan Marušič ${ }^{\dagger}$ \\ University of Primorska, FAMNIT, Glagoljaška 8, 6000 Koper, Slovenia \\ and \\ University of Ljubljana, IMFM, Jadranska 19, 1111 Ljubljana, Slovenia \\ Štefko Miklavič \\ University of Primorska, FAMNIT, Glagoljaška 8, 6000 Koper, Slovenia
}

Received 15 July 2008, accepted 1 January 2009, published online 13 March 2009

\begin{abstract}
A graph $X$ is said to be strongly distance-balanced whenever for any edge $u v$ of $X$ and any positive integer $i$, the number of vertices at distance $i$ from $u$ and at distance $i+1$ from $v$ is equal to the number of vertices at distance $i+1$ from $u$ and at distance $i$ from $v$. It is proven that for any integers $k \geq 2$ and $n \geq k^{2}+4 k+1$, the generalized Petersen graph $\mathrm{GP}(n, k)$ is not strongly distance-balanced.
\end{abstract}

Keywords: Graph, strongy distance-balanced, generalized Petersen graph.

Math. Subj. Class.: 05C07, 05C12

*Supported in part by "Agencija za raziskovalno dejavnost Republike Slovenije", research program P1-0285

${ }^{\dagger}$ Corresponding author.

E-mail addresses: klavdija.kutnar@upr.si (Klavdija Kutnar), aleksander.malnic@guest.arnes.si (Aleksander Malnič), dragan.marusic@upr.si (Dragan Marušič), stefko.miklavic@upr.si (Štefko Miklavič) 


\section{Introduction}

Let $X$ be a graph with diameter $d$, and let $V(X)$ and $E(X)$ denote the vertex set and the edge set of $X$, respectively. For $u, v \in V(X)$, we let $d(u, v)$ denote the minimal pathlength distance between $u$ and $v$. We say that $X$ is distance-balanced whenever for an arbitrary pair of adjacent vertices $u$ and $v$ of $X$

$$
|\{x \in V(X) \mid d(x, u)<d(x, v)\}|=|\{x \in V(X) \mid d(x, v)<d(x, u)\}|
$$

holds. These graphs were, at least implicitly, first studied by Handa [1] who considered distance-balanced partial cubes. The term itself, however, is due to Jerebic, Klavžar and Rall [3] who studied distance-balanced graphs in the framework of various kinds of graph products.

Let $u v$ be an arbitrary edge of $X$. For any two nonnegative integers $i, j$, we let

$$
D_{j}^{i}(u, v)=\{x \in V(X) \mid d(u, x)=i \text { and } d(v, x)=j\} .
$$

The triangle inequality implies that only the sets $D_{i}^{i-1}(u, v), D_{i}^{i}(u, v)$ and $D_{i-1}^{i}(u, v)$ $(1 \leq i \leq d)$ can be nonempty. One can easily see that $X$ is distance-balanced if and only if for every edge $u v \in E(X)$

$$
\sum_{i=1}^{d}\left|D_{i-1}^{i}(u, v)\right|=\sum_{i=1}^{d}\left|D_{i}^{i-1}(u, v)\right|
$$

holds.

Obviously, if $\left|D_{i-1}^{i}(u, v)\right|=\left|D_{i}^{i-1}(u, v)\right|$ holds for $1 \leq i \leq d$ and for every edge $u v \in E(X)$, then $X$ is distance-balanced. The converse, however, is not necessarily true. For instance, in the generalized Petersen graphs $\operatorname{GP}(24,4), \operatorname{GP}(35,8)$ and $\operatorname{GP}(35,13)$ (see Section 2 for the definition of generalized Petersen graphs), we can find two adjacent vertices $u, v$ and an integer $i$, such that $\left|D_{i-1}^{i}(u, v)\right| \neq\left|D_{i}^{i-1}(u, v)\right|$. But it is easy to see that these graphs are distance-balanced.

We therefore say that $X$ is strongly distance-balanced, if $\left|D_{i-1}^{i}(u, v)\right|=\left|D_{i}^{i-1}(u, v)\right|$ for every positive integer $i$ and every edge $u v \in E(X)$. Let us remark that graphs with this property are also called distance-degree regular. Distance-degree regular graphs were studied in [2].

For a graph $X$, a vertex $u$ of $X$ and an integer $i$, let $S_{i}(u)=\{x \in V(X) \mid d(x, u)=i\}$ denote the set of vertices of $X$ which are at distance $i$ from $u$. The following result was proven in [4].

Proposition 1.1. [4, Proposition 2.1] Let $X$ be a graph with diameter $d$. Then $X$ is strongly distance-balanced if and only if $\left|S_{i}(u)\right|=\left|S_{i}(v)\right|$ holds for every edge uv $\in E(X)$ and every $i \in\{0, \ldots, d\}$.

In [3], the following conjecture was stated.

Conjecture 1.2. [3, Conjecture 2.5] For any integer $k \geq 2$ there exists a positive integer $n_{0}$ such that the generalized Petersen graph $\mathrm{GP}(n, k)$ is not distance-balanced for every integer $n \geq n_{0}$.

In this short note we prove the following slightly weaker result. 
Theorem 1.3. For any integers $k \geq 2$ and $n \geq k^{2}+4 k+1$, the generalized Petersen graph $\mathrm{GP}(n, k)$ is not strongly distance-balanced.

We will prove Theorem 1.3 in two steps. In the first step we prove that the graph $\mathrm{GP}\left(k^{2}+4 k+1, k\right)$ is not strongly distance-balanced. In the second step we use the result from the first step to prove that $\operatorname{GP}(n, k)$ is not strongly distance-balanced if $n \geq$ $k^{2}+4 k+1$.

\section{Proof of Theorem 1.3}

Let $n \geq 3$ be a positive integer, and let $k \in\{1, \ldots, n-1\} \backslash\{n / 2\}$. The generalized Petersen graph $\operatorname{GP}(n, k)$ is defined to have the following vertex set and edge set:

$$
\begin{aligned}
& V(\operatorname{GP}(n, k))=\left\{u_{i} \mid i \in \mathbb{Z}_{n}\right\} \cup\left\{v_{i} \mid i \in \mathbb{Z}_{n}\right\}, \\
& E(\operatorname{GP}(n, k))=\left\{u_{i} u_{i+1} \mid i \in \mathbb{Z}_{n}\right\} \cup\left\{v_{i} v_{i+k} \mid i \in \mathbb{Z}_{n}\right\} \cup\left\{u_{i} v_{i} \mid i \in \mathbb{Z}_{n}\right\} .
\end{aligned}
$$

Note that $\operatorname{GP}(n, k)$ is cubic, and that it is bipartite precisely when $n$ is even and $k$ is odd. It is easy to see that $\operatorname{GP}(n, k) \cong \operatorname{GP}(n, n-k)$. Furthermore, if the multiplicative inverse $k^{-1}$ of $k$ exists in $\mathbb{Z}_{n}$, then the mapping $f: V(\operatorname{GP}(n, k)) \rightarrow V\left(\operatorname{GP}\left(n, k^{-1}\right)\right)$ defined by the rule

$$
f\left(u_{i}\right)=v_{k^{-1} i}, \quad f\left(v_{i}\right)=u_{k^{-1} i}
$$

gives rise to an isomorphism of graphs $\operatorname{GP}(n, k)$ and $\operatorname{GP}\left(n, k^{-1}\right)$, where the use of the same symbols for vertices in $\operatorname{GP}(n, k)$ and $\operatorname{GP}\left(n, k^{-1}\right)$ should cause no confusion.

We first investigate the sets $S_{i}\left(u_{0}\right)$ and $S_{i}\left(v_{0}\right)$ of the graph $\operatorname{GP}\left(k^{2}+4 k+1, k\right)$.

Lemma 2.1. Let $k \geq 9$ be an integer, let $n=k^{2}+4 k+1$ and let $u_{0} \in V(G P(n, k))$. Then the following statements hold:

(i) $S_{1}\left(u_{0}\right)=\left\{u_{ \pm 1}, v_{0}\right\}, S_{2}\left(u_{0}\right)=\left\{u_{ \pm 2}, v_{ \pm 1}, v_{ \pm k}\right\}$,

$$
S_{3}\left(u_{0}\right)=\left\{u_{ \pm 3}, u_{ \pm k}, v_{ \pm 2}, v_{ \pm(k+1)}, v_{ \pm(k-1)}, v_{ \pm 2 k}\right\} \text {; }
$$

(ii) if $i \in\{4, \ldots,\lfloor k / 2\rfloor+1\}$, then

$$
\begin{aligned}
S_{i}\left(u_{0}\right)=\{ & \left.u_{ \pm i}, u_{ \pm(i-2) k}\right\} \cup\left\{v_{ \pm(i-1)}, v_{ \pm(i-1) k}\right\} \cup \\
& \left\{u_{ \pm(l k+i-l-2)}, u_{ \pm(l k-i+l+2)} \mid 1 \leq l \leq i-3\right\} \cup \\
& \left\{v_{ \pm(l k+i-l-1)}, v_{ \pm(l k-i+l+1)} \mid 1 \leq l \leq i-2\right\}
\end{aligned}
$$

(iii) if $k$ is odd, then

$$
\begin{aligned}
S_{(k+3) / 2}\left(u_{0}\right)=\{ & \left.u_{ \pm(k+3) / 2}, u_{ \pm(k-1) k / 2}, u_{ \pm(3 k-3) / 2}\right\} \cup \\
& \left\{u_{ \pm(l k+(k-1) / 2-l)}, u_{ \pm(l k-(k-1) / 2+l)} \mid 2 \leq l \leq(k-3) / 2\right\} \cup \\
& \left\{v_{ \pm(k+1) / 2}, v_{ \pm(k+1) k / 2}, v_{ \pm(3 k-1) / 2}\right\} \cup \\
& \left\{v_{ \pm(l k+(k+1) / 2-l)}, v_{ \pm(l k-(k+1) / 2+l)} \mid 2 \leq l \leq(k-1) / 2\right\} ;
\end{aligned}
$$

(iv) if $k$ is even, then

$$
\begin{aligned}
S_{(k+4) / 2}\left(u_{0}\right)=\{ & \left.u_{ \pm k^{2} / 2}, u_{ \pm(3 k-2) / 2}\right\} \cup \\
& \left\{u_{ \pm(l k+k / 2-l)}, u_{ \pm(l k-k / 2+l)} \mid 2 \leq l \leq(k-2) / 2\right\} \cup \\
& \left\{v_{ \pm 3 k / 2}, v_{ \pm(k+2) k / 2}\right\} \cup \\
& \left\{v_{ \pm(l k+3 k / 2-l)}, v_{ \pm(l k+k / 2+l)} \mid 1 \leq l \leq(k-2) / 2\right\} .
\end{aligned}
$$


Proof. Using the fact that by assumption $k \geq 9$, a careful inspection of the neighbors' sets of vertices $u_{i}$ and $v_{i}$, we see that (i) holds.

We now prove part (ii) by induction. Similarly as above we see that (ii) holds for $i \in\{4,5\}$.

Let us now assume that (ii) holds for $i-1$ and $i$, where $i \in\{5, \ldots,\lfloor k / 2\rfloor\}$. Hence we have

$$
\begin{array}{r}
S_{i-1}\left(u_{0}\right)=\left\{u_{ \pm(i-1)}, u_{ \pm(i-3) k}\right\} \cup\left\{u_{ \pm(l k+i-l-3)}, u_{ \pm(l k-i+l+3)} \mid 1 \leq l \leq i-4\right\} \cup \\
\left\{v_{ \pm(i-2)}, v_{ \pm(i-2) k}\right\} \cup\left\{v_{ \pm(l k+i-l-2)}, v_{ \pm(l k-i+l+2)} \mid 1 \leq l \leq i-3\right\}
\end{array}
$$

and

$$
\begin{aligned}
S_{i}\left(u_{0}\right)=\left\{u_{ \pm i}, u_{ \pm(i-2) k}\right\} \cup\left\{u_{ \pm(l k+i-l-2)}, u_{ \pm(l k-i+l+2)} \mid 1 \leq l \leq i-3\right\} \cup \\
\left\{v_{ \pm(i-1)}, v_{ \pm(i-1) k}\right\} \cup\left\{v_{ \pm(l k+i-l-1)}, v_{ \pm(l k-i+l+1)} \mid 1 \leq l \leq i-2\right\} .
\end{aligned}
$$

Now we compute the neighbors of the vertices belonging to the set $S_{i}\left(u_{0}\right)$. Since

$$
\begin{aligned}
& S_{1}\left(u_{-r}\right)=\left\{u_{-q}, v_{-q} \mid u_{q}, v_{q} \in S_{1}\left(u_{r}\right)\right\} \quad \text { and } \\
& S_{1}\left(v_{-r}\right)=\left\{u_{-q}, v_{-q} \mid u_{q}, v_{q} \in S_{1}\left(v_{r}\right)\right\},
\end{aligned}
$$

we will only list the following sets:

- $S_{1}\left(u_{i}\right)=\left\{u_{i+1}, u_{i-1}, v_{i}\right\}$,

- $S_{1}\left(u_{(i-2) k}\right)=\left\{u_{(i-2) k+(i+1)-(i-2)-2}, u_{(i-2) k-(i+1)+(i-2)+2}, v_{(i-2) k}\right\}$,

- $S_{1}\left(u_{l k+i-l-2}\right)=\left\{u_{l k+(i+1)-l-2}, u_{l k+(i-1)-l-2}, v_{l k+(i-1)-l-1}\right\}$,

- $S_{1}\left(u_{l k-i+l+2}\right)=\left\{u_{l k-(i-1)+l+2}, u_{l k-(i+1)+l+2}, v_{l k-(i-1)+l+1}\right\}$,

- $S_{1}\left(v_{i-1}\right)=\left\{u_{i-1}, v_{k+(i+1)-2}, v_{-(k-(i+1)+2)}\right\}$,

- $S_{1}\left(v_{(i-1) k}\right)=\left\{u_{(i-1) k}, v_{i k}, v_{(i-2) k}\right\}$,

- $S_{1}\left(v_{l k+i-l-1}\right)=\left\{u_{l k+(i+1)-l-2}, v_{(l+1) k+(i+1)-(l+1)-1}, v_{(l-1) k+(i-1)-(l-1)-1}\right\}$,

- $S_{1}\left(v_{l k-i+l+1}\right)=\left\{u_{l k-(i+1)+l+2}, v_{(l+1) k-(i+1)+(l+1)+1}, v_{(l-1) k-(i-1)+(l-1)+1}\right\}$.

Obviously, $S_{i+1}\left(u_{0}\right)$ consists of all the neighbors of vertices in $S_{i}\left(u_{0}\right)$, which are not in $S_{i-1}\left(u_{0}\right)$ or $S_{i}\left(u_{0}\right)$. Thus

$$
\begin{aligned}
S_{i+1}\left(u_{0}\right)=\{ & \left.u_{ \pm(i+1)}, u_{ \pm(i-1) k}\right\} \cup \\
& \left\{u_{ \pm(l k+(i+1)-l-2)}, u_{ \pm(l k-(i+1)+l+2)} \mid 1 \leq l \leq i-2\right\} \cup \\
\quad & \quad\left\{v_{ \pm i}, v_{ \pm i k}\right\} \cup\left\{v_{ \pm(l k+(i+1)-l-1)}, v_{ \pm(l k-(i+1)+l+1)} \mid 1 \leq l \leq i-1\right\}
\end{aligned}
$$

and the result follows.

Let us now prove (iii). Assume first $k$ is odd, and abbreviate $b=(k+1) / 2$. By (ii),

$$
\begin{array}{r}
S_{b-1}\left(u_{0}\right)=\left\{u_{ \pm(b-1)}, u_{ \pm(b-3) k}\right\} \cup\left\{u_{ \pm(l k+b-l-3)}, u_{ \pm(l k-b+l+3)} \mid 1 \leq l \leq b-4\right\} \cup \\
\left\{v_{ \pm(b-2)}, v_{ \pm(b-2) k}\right\} \cup\left\{v_{ \pm(l k+b-l-2)}, v_{ \pm(l k-b+l+2)} \mid 1 \leq l \leq b-3\right\}
\end{array}
$$


and

$$
\begin{aligned}
S_{b}\left(u_{0}\right)=\left\{u_{ \pm b}, u_{ \pm(b-2) k}\right\} \cup\left\{u_{ \pm(l k+b-l-2)}, u_{ \pm(l k-b+l+2)} \mid 1 \leq l \leq b-3\right\} \cup \\
\left\{v_{ \pm(b-1)}, v_{ \pm(b-1) k}\right\} \cup\left\{v_{ \pm(l k+b-l-1)}, v_{ \pm(l k-b+l+1)} \mid 1 \leq l \leq b-2\right\} .
\end{aligned}
$$

Let us now compute the neighbors of the vertices in $S_{b}\left(u_{0}\right)$. Since $S_{1}\left(u_{-r}\right)=\left\{u_{-q}, v_{-q} \mid\right.$ $\left.u_{q}, v_{q} \in S_{1}\left(u_{r}\right)\right\}$ and $S_{1}\left(v_{-r}\right)=\left\{u_{-q}, v_{-q} \mid u_{q}, v_{q} \in S_{1}\left(v_{r}\right)\right\}$, we will only list the following sets:

- $S_{1}\left(u_{b}\right)=\left\{u_{b+1}, u_{b-1}, v_{b}\right\}$,

- $S_{1}\left(u_{(b-2) k}\right)=\left\{u_{(b-2) k+(b+1)-(b-2)-2}, u_{(b-2) k-(b+1)+(b-2)+2}, v_{(b-2) k}\right\}$,

- $S_{1}\left(u_{l k+b-l-2}\right)=\left\{u_{l k+b-l-1}, u_{l k+b-l-3}, v_{l k+b-l-2}\right\}$,

- $S_{1}\left(u_{l k-b+l+2}\right)=\left\{u_{l k-b+l+3}, u_{l k-b+l+1}, v_{l k-b+l+2}\right\}$,

- $S_{1}\left(v_{b-1}\right)=\left\{u_{b-1}, v_{k+b-1}, v_{-(k-b+1)}\right\}=\left\{u_{b-1}, v_{k+b-1}, v_{-b}\right\}$,

- $S_{1}\left(v_{(b-1) k}\right)=\left\{u_{(b-1) k}, v_{b k}, v_{(b-2) k}\right\}$,

- $S_{1}\left(v_{l k+b-l-1}\right)=\left\{u_{l k+b-l-1}, v_{(l+1) k+b-l-1}, v_{(l-1) k+b-l-1}\right\}$,

- $S_{1}\left(v_{l k-b+l+1}\right)=\left\{u_{l k-b+l+1}, v_{(l+1) k-b+l+1}, v_{(l-1) k-b+l+1}\right\}$.

Observe that $u_{ \pm(k-b+2)}=u_{ \pm(b+1)}$. Therefore, sorting out those neigbors of the vertices in $S_{b}\left(u_{0}\right)$ which are either in $S_{b-1}\left(u_{0}\right)$ or $S_{b}\left(u_{0}\right)$, we obtain that

$$
\begin{aligned}
S_{b+1}\left(u_{0}\right)=\{ & \left.u_{ \pm(b+1)}, u_{ \pm(b-1) k}, u_{ \pm(k+b-2)}\right\} \cup \\
& \left\{u_{ \pm(l k+b-l-1)}, u_{ \pm(l k-b+l+1)} \mid 2 \leq l \leq b-2\right\} \cup \\
& \left\{v_{ \pm b}, v_{ \pm b k}, v_{ \pm(k+b-1)}\right\} \cup\left\{v_{ \pm(l k+b-l)}, v_{ \pm(l k-b+l)} \mid 2 \leq l \leq b-1\right\}
\end{aligned}
$$

and hence the result follows.

The proof of (iv) is done in a similar way to that of (iii) above and is omitted.

We have the following immediate corollary of Lemma 2.1.

Corollary 2.2. Let $k \geq 9$ be an integer, let $n=k^{2}+4 k+1$ and let $u_{0} \in V(G P(n, k))$. Then the following statements hold:

(i) $\left|S_{1}\left(u_{0}\right)\right|=3,\left|S_{2}\left(u_{0}\right)\right|=6,\left|S_{3}\left(u_{0}\right)\right|=12$;

(ii) $\left|S_{i}\left(u_{0}\right)\right|=8 i-12$ for $i \in\{4, \ldots,\lfloor k / 2\rfloor+1\}$;

(iii) if $k$ is odd, then $\left|S_{(k+3) / 2}\left(u_{0}\right)\right|=4 k-4$;

(iv) if $k$ is even, then $\left|S_{(k+4) / 2}\left(u_{0}\right)\right|=4 k-4$.

The proofs of the next lemma and corollary are omitted as they can be carried out using the same arguments as in the proof of Lemma 2.1. (Note that $-(k+4)$ is the multiplicative inverse of $k$ in $\mathbb{Z}_{k^{2}+4 k+1}$.)

Lemma 2.3. Let $k \geq 9$ be an integer, let $n=k^{2}+4 k+1$, and let $u_{0} \in V(G P(n, k+4))$. Then the following statements hold:

(i) $S_{1}\left(u_{0}\right)=\left\{u_{ \pm 1}, v_{0}\right\}, S_{2}\left(u_{0}\right)=\left\{u_{ \pm 2}, v_{ \pm 1}, v_{ \pm(k+4)}\right\}$, $S_{3}\left(u_{0}\right)=\left\{u_{ \pm 3}, u_{ \pm(k+4)}, v_{ \pm 2}, v_{ \pm(k+5)}, v_{ \pm(k+3)}, v_{ \pm 2(k+4)}\right\}$; 
(ii) if $i \in\{4, \ldots,\lfloor k / 2\rfloor+1\}$, then

$$
\begin{aligned}
S_{i}\left(u_{0}\right)=\{ & \left.u_{ \pm i}, u_{ \pm(i-2)(k+4)}\right\} \cup\left\{v_{ \pm(i-1)}, v_{ \pm(i-1)(k+4)}\right\} \cup \\
& \left\{u_{ \pm(l k+i+3 l-2)}, u_{ \pm(l k-i+5 l+2)} \mid 1 \leq l \leq i-3\right\} \cup \\
& \left\{v_{ \pm(l k+i+3 l-1)}, v_{ \pm(l k-i+5 l+1)} \mid 1 \leq l \leq i-2\right\}
\end{aligned}
$$

(iii) if $k$ is odd, then

$$
\begin{aligned}
S_{(k+3) / 2}\left(u_{0}\right)=\{ & \left.u_{ \pm(k+3) / 2}, u_{ \pm(k-1)(k+4) / 2}\right\} \cup \\
& \left\{u_{ \pm(l k+(k-1) / 2+3 l)}, u_{ \pm(l k-(k-1) / 2+5 l)} \mid 1 \leq l \leq(k-3) / 2\right\} \cup \\
& \left\{v_{ \pm(k+1) / 2}, v_{ \pm(k+1)(k+4) / 2}, v_{ \pm\left(k^{2}+3 k-6\right) / 2}\right\} \cup \\
& \left\{v_{ \pm(l k+(k+1) / 2+3 l)}, v_{ \pm(l k-(k+1) / 2+5 l)} \mid 1 \leq l \leq(k-3) / 2\right\} ;
\end{aligned}
$$

(iv) if $k$ is even, then

$$
\begin{aligned}
S_{(k+4) / 2}\left(u_{0}\right)=\{ & \left.u_{ \pm(k+4) / 2}, u_{ \pm k(k+4) / 2}\right\} \cup \\
& \left\{u_{ \pm(l k+k / 2+3 l)}, u_{ \pm(l k-k / 2+5 l)} \mid 1 \leq l \leq(k-2) / 2\right\} \cup \\
& \left\{v_{ \pm(k+2) / 2}, v_{ \pm(k+2)^{2} / 2}\right\} \cup \\
& \left\{v_{ \pm(l k+(k+2) / 2+3 l)}, v_{ \pm(l k-(k+2) / 2+5 l)} \mid 1 \leq l \leq(k-2) / 2\right\} .
\end{aligned}
$$

Corollary 2.4. Let $k \geq 9$ be an integer, let $n=k^{2}+4 k+1$ and let $u_{0} \in V(G P(n, k+4))$. Then the following statements hold:

(i) $\left|S_{1}\left(u_{0}\right)\right|=3,\left|S_{2}\left(u_{0}\right)\right|=6,\left|S_{3}\left(u_{0}\right)\right|=12$;

(ii) $\left|S_{i}\left(u_{0}\right)\right|=8 i-12$ for $i \in\{4, \ldots,\lfloor k / 2\rfloor+1\}$;

(iii) if $k$ is odd, then $\left|S_{(k+3) / 2}\left(u_{0}\right)\right|=4 k-2$;

(iv) if $k$ is even, then $\left|S_{(k+4) / 2}\left(u_{0}\right)\right|=4 k$.

Corollary 2.5. Let $k \geq 2$ be an integer, let $n=k^{2}+4 k+1$, let $b=\lfloor k / 2\rfloor+2$ and let $u_{0}, v_{0} \in V(G P(n, k))$. Then $\left|S_{b}\left(u_{0}\right)\right| \neq\left|S_{b}\left(v_{0}\right)\right|$. In particular, $G P(n, k)$ is not strongly distance-balanced.

Proof. If $k \leq 8$, then a direct check shows that $\left|S_{b}\left(u_{0}\right)\right| \neq\left|S_{b}\left(v_{0}\right)\right|$. Assume now $k \geq 9$. Note that $-(k+4)=n-(k+4) \in \mathbb{Z}_{n}$ is the multiplicative inverse of $k \in \mathbb{Z}_{n}$. Therefore, by (2.2), we have

$$
\mathrm{GP}(n,(k+4)) \cong \mathrm{GP}(n,-(k+4)) \cong \mathrm{GP}(n, k) .
$$

Under this isomorphism, the vertex $u_{0} \in V(\mathrm{GP}(n,(k+4)))$ maps to the vertex $v_{0} \in$ $V(\operatorname{GP}(n, k))$. (Recall that the same symbols are used for vertices in $\operatorname{GP}(n, k)$ and in $\operatorname{GP}(n,(k+4))$.) The result now follows from Corollaries 2.2 and 2.4.

We are now ready to prove our main result.

Proof of Theorem 1.3. Let $k \geq 2$ be an integer, let $n_{0}=k^{2}+4 k+1$, let $n \geq n_{0}$, and let $b=\lfloor k / 2\rfloor+2$. We now show that $\operatorname{GP}(n, k)$ is not strongly distance-balanced. In what follows, the same symbols are used for vertices in $\operatorname{GP}\left(n_{0}, k\right)$ and those in $\operatorname{GP}(n, k)$.

Observe that $k b<n_{0} / 2$. By (2.1), for $i \in\{1,2, \ldots, b\}$ we have that $u_{j} \in V(\operatorname{GP}(n, k))$ $\left(v_{j} \in V(\operatorname{GP}(n, k))\right.$, respectively) is at distance $i$ from $u_{0} \in V(\operatorname{GP}(n, k))$ if and only if $u_{j} \in V\left(\operatorname{GP}\left(n_{0}, k\right)\right)\left(v_{j} \in V\left(\operatorname{GP}\left(n_{0}, k\right)\right)\right.$, respectively) is at distance $i$ from $u_{0} \in$ $V\left(\operatorname{GP}\left(n_{0}, k\right)\right)$. Therefore, the number of vertices which are at distance $i$ from $u_{0} \in$ 
$V(\operatorname{GP}(n, k))$ is the same as the number of vertices which are at distance $i$ from $u_{0} \in$ $V\left(\operatorname{GP}\left(n_{0}, k\right)\right)$. Similarly, for $i \in\{1,2, \ldots, b\}$, we have that $u_{j} \in V(\operatorname{GP}(n, k))\left(v_{j} \in\right.$ $V(\operatorname{GP}(n, k))$, respectively) is at distance $i$ from $v_{0} \in V(\operatorname{GP}(n, k))$ if and only if $u_{j} \in$ $V\left(\operatorname{GP}\left(n_{0}, k\right)\right)\left(v_{j} \in V\left(\operatorname{GP}\left(n_{0}, k\right)\right)\right.$, respectively) is at distance $i$ from $v_{0} \in V\left(\operatorname{GP}\left(n_{0}, k\right)\right)$. Hence the number of vertices which are at distance $i$ from the vertex $v_{0} \in V(\operatorname{GP}(n, k))$ is the same as the number of vertices which are at distance $i$ from the vertex $v_{0} \in V\left(\operatorname{GP}\left(n_{0}\right.\right.$, $k)$ ). Therefore, by Corollary 2.5, $\left|S_{b}\left(u_{0}\right)\right| \neq\left|S_{b}\left(v_{0}\right)\right|$ for $u_{0}, v_{0} \in V(\operatorname{GP}(n, k))$. By Proposition 1.1, $\operatorname{GP}(n, k)$ is not strongly distance-balanced.

\section{References}

[1] K. Handa, Bipartite graphs with balanced ( $a, b)$-partitions, Ars Combin. 51 (1999), 113-119.

[2] T. Hilado and K. Nomura, Distance Degree Regular Graphs, J. Combin. Theory B 37 (1984), 96-100.

[3] J. Jerebic, S. Klavžar, D. F. Rall, Distance-balanced graphs, Ann. Combin. 12 (2008), 71-79.

[4] K. Kutnar, A. Malnič, D. Marušič, Š. Miklavič, Distance-balanced graphs: symmetry conditions, Discrete Math. 306 (2006), 1881-1894. 